\title{
Gait Recognition Based on Outermost Contour
}

\author{
Lili Liu, Yilong Yin *, Wei Qin, Ying Li \\ School of Computer Science and Technology, Shandong University, \\ Jinan, 250101, China \\ ll_liu@yahoo.com.cn \\ ylyin@sdu.edu.cn \\ qinwei1130@yahoo.cn \\ liying200606@163.com
}

\begin{abstract}
Gait recognition aims to identify people by the way they walk. In this paper, a simple but effective gait recognition method based on outermost contour is proposed. For each gait image sequence, an adaptive silhouette extraction algorithm is firstly used to segment the frames of the sequence and a series of postprocessing is applied to obtain the normalized silhouette images with less noise. Then a novel feature extraction method based on outermost contour is performed. Principal Component Analysis (PCA) is adopted to reduce the dimensionality of the distance signals derived from the outermost contours of silhouette images. Then Multiple Discriminant Analysis (MDA) is used to optimize the separability of gait features belonging to different classes. Nearest Neighbor (NN) classifier and Nearest Neighbor classifier with respect to class Exemplars (ENN) are used to classify the final feature vectors produced by MDA. In order to verify the effectiveness and robustness of our feature extraction algorithm, we also use two other classifiers - Backpropagation Neural Network (BPNN) and Support Vector Machine (SVM) for recognition. Experimental results on a gait database of 100 people show that the accuracy of using MDA, BPNN and SVM can achieve $97.67 \%, 94.33 \%$ and $94.67 \%$, respectively.
\end{abstract}

Keywords: Gait recognition, Outermost Contour, Principal Component Analysis, Multiple Discriminant Analysis, Back Propagation Neural Network, Support Vector Machine.

\section{Introduction}

Gait recognition, aiming to identify individuals by the way they walk, is a relatively new research direction in biometrics. In comparison with the first generation biometric traits such as fingerprint, face and iris, gait has many advantages. It does not require users' interaction and it is non-invasive. Also it is difficult to conceal or disguise. Furthermore, gait can be effective for recognition at a distance or at low resolution, while other biometric traits are not available. To the best of our knowledge, gait is the only perceivable biometric trait from a great distance. Therefore, gait receives increasing interest from researchers and various approaches have been proposed on gait recognition domain recently.

Current gait recognition approaches can be divided into two categories: model-based ones and model-free ones. Model-based approaches construct

*Corresponding Author. Tel./ Fax: +86-531-88391367 
human model and use the parameters of the model for recognition. An early such attempt ${ }^{1}$ modeled the lower limbs as two inter-connected pendulum. Lee and Grimson ${ }^{2}$ used seven ellipses to model the human body. Yam et al. $^{3}$ used double pendulum to describe the thigh and lower leg movements. However, the majority of current approaches are the model-free approaches which are simple and fast. The model-free approaches do not model the structure of human motion, but deal directly with image statistics. Murase and Sakai ${ }^{4}$ presented a template matching method which used the parametric eigenspace representation to reduce the computational cost. Little and Boyd ${ }^{5}$ used scaleindependent features from moments of the dense optical flow to represent the shape of human motion. Wang et al. ${ }^{6}$ extracted gait feature through upwrapping the outer contour of each silhouette. Han et al. ${ }^{7}$ represented gait image sequence by gait energy image and synthetic template, and used fused feature for recognition. Chen et al. ${ }^{8}$ proposed frame difference energy image to suppress the influence of silhouette incompleteness in gait recognition.

All the proposed approaches promote the development of gait recognition domain. However, there are still many challenges in gait recognition, such as imperfect segmentation of the walking subject, different walking directions of the subject, changes in clothes, and changes of gait as a result of mood or injury, or as a result of objects carrying. In this paper, we propose a model-free gait recognition approach which can tolerate imperfect segmentation to some extent.

In fact, this paper is an extension of an earlier version presented in paper ${ }^{9}$. The main contribution of paper ${ }^{9}$ is a novel gait feature extraction method based on outermost contour. This method is easy to comprehend and implement, and has a very low computational cost. Based on this contribution, we make two main extensions in current paper. The two main extensions are summarized as follows:

- In order to verify the effectiveness and the robustness of the proposed feature extraction method, we introduce other two classifiers - BPNN and SVM for recognition.

- We carry out comparisons on recognition accu- racy between our method and other state-of-theart gait recognition methods.

The overview of our gait recognition method is shown in Fig. 1. It contains two major parts - the training part and the testing part. In training part, we first extract features from the input training gait sequences. Then PCA is performed to reduce the dimensionality of the extracted features. Finally, one of the three classification approaches - MDA, BPNN and SVM is used for training. In testing part, we also extract feature of the test gait sequence firstly. Then the model established in the training stage is used to identify the feature of the testing gait sequence.

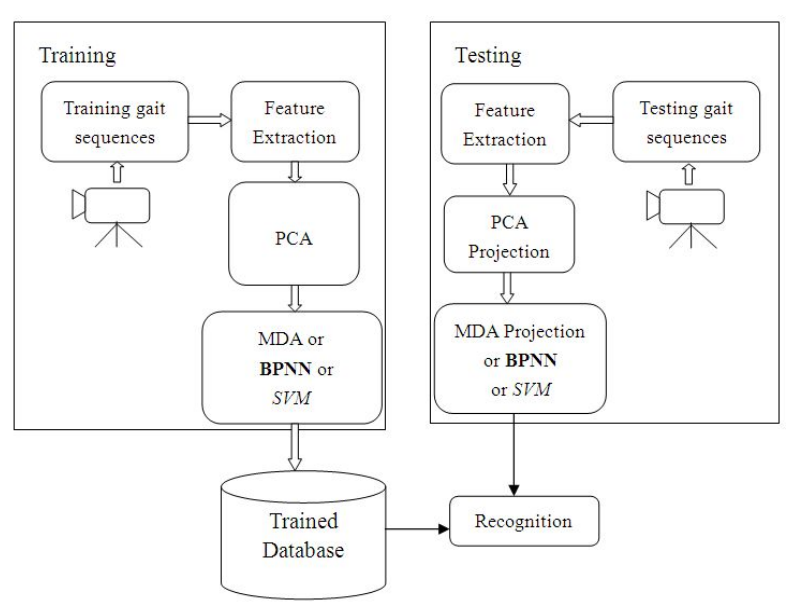

Fig. 1. Overview of our gait recognition method.

The remainder of this paper is organized as follows: Section 2 describes the proposed feature extraction method in detail. In section 3, we give a brief introduction of the three classification approaches - MDA with NN or ENN, BPNN and SVM. Experimental results are presented in Section 4 , and Section 5 gives conclusions of the paper.

\section{Feature Extraction}

In this section, we first introduce the silhouette segmentation and preprocessing methods, and then present the proposed silhouette representation method. Finally, we describe the method to obtain gait feature of each gait sequence. 


\subsection{Preprocessing}

Silhouette segmentation is the first step to gait recognition. We apply an existing adaptive gait silhouette extraction algorithm using Gauss model proposed by $\mathrm{Fu}^{10}$ to extract the walking subject for better segmentation performance. Then for each binary silhouette image, we use morphological operators such as dilation and erosion to fill the small holes inside the silhouette and to filter small noises on the background area. A binary connected component analysis is finally applied to extract the connected region with the largest size for ignoring all the remaining noises.

In consideration of the convenience of the following silhouette representation and time consumption, we normalize the silhouette images to the same size (Proportionally resize each silhouette image to make all the silhouettes have the same height, and align the normalized silhouette to the horizontal center.). Every image was resized to $128 \times 100$ pixels in this paper. It is to be noted that the height of each silhouette is also 100 pixels. An example of silhouette segmentation is shown in Fig. 2, from which we can see that the silhouette segmentation procedure performs well as a whole.

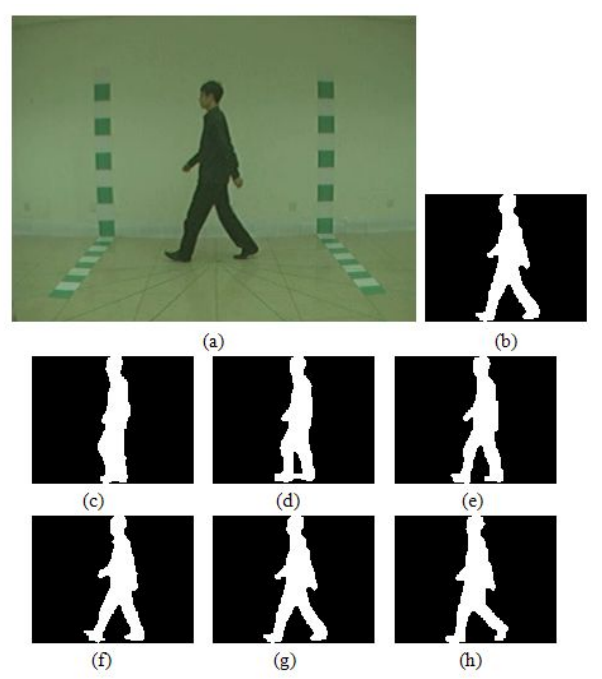

Fig. 2. An example of silhouette segmentation: (a) an original image in gait database, (b) the normalized and aligned silhouette of (b), (c)-(h) temporal changes of six successive frames in a gait silhouette sequence.

\subsection{Silhouette Representation}

In a gait silhouette sequence, the only cue to identify the gait depends on temporal changes of the silhouette. In order to reduce the computational cost, we propose a new silhouette representation method, which only uses some of the pixels on the contour, to describe the temporal changes of the silhouette. For the sake of description, we make a definition as follows:

Outermost contour: In each row of a normalized silhouette image, the most right pixel and the most left pixel on the contour belong to outermost contour. Fig. 3(a) shows the schematic of outermost contour. The bold boundaries in Fig. 3(a) belong to the outermost contour, but the thin boundary between the two legs does not belong to the outmost contour. Because all the silhouettes are normalized, the number of pixels on the outermost contour is definite (i.e., $2 H$ where $H$ is the height of the silhouette measured in pixels).

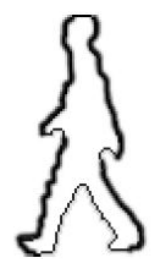

(a)

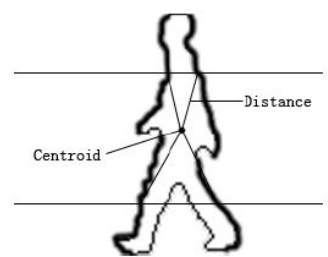

(b)
Fig. 3. (a) the schematic of outermost contour, (b) illustration of distance signal extraction.

Firstly, we compute the centroid $\left(x_{c}, y_{c}\right)$ of the outermost contour.

$$
\begin{aligned}
& x_{c}=\frac{1}{n} \sum_{i=1}^{n} x_{i}, \\
& y_{c}=\frac{1}{n} \sum_{i=1}^{n} y_{i}
\end{aligned}
$$

where $n$ is the number of pixels on the outermost contour, $\left(x_{i}, y_{i}\right)$ is the coordinate of pixel on the outermost contour. Actually, $n=2 H$ as mentioned above.

Secondly, we compute the distance between each outermost contour pixel $\left(x_{i}, y_{i}\right)$ and the centroid $\left(x_{c}, y_{c}\right)$ row by row, as is shown in Fig. 3(b). 


$$
d_{i}=\sqrt{\left(x_{i}-x_{c}\right)^{2}+\left(y_{i}-y_{c}\right)^{2}}
$$

Thus, for each silhouette image, we obtain a distance signal $D=\left[d_{1}, d_{2}, \cdots, d_{2 H}\right]$ which is composed of all the distances $d_{i}$.

Compared with the silhouette representation method ${ }^{6}$ which needs unwrap the outer contour and normalize the computed distance signals, our silhouette representation method based on outermost contour is simpler and easier to implement, and has lower computational cost. Besides, the proposed silhouette representation method ignores the region between two legs where imperfect segmentation often exists as a result of the shadow of legs, which is good for recognition. Four images of this kind of imperfect segmentation are shown in Fig. 4.

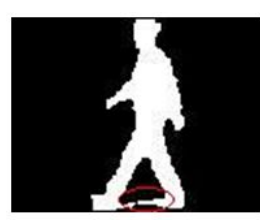

(a)

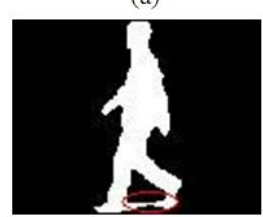

(c)

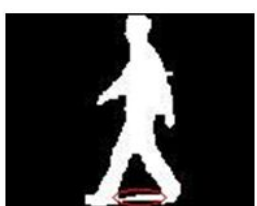

(b)

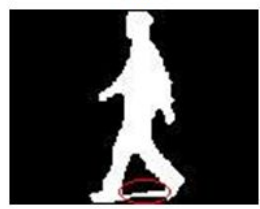

(d)
Fig. 4. Four images with imperfect segmentation.

\subsection{Gait Feature}

Although we have enormously reduced the dimensionality of the silhouette image in Section 2.2, the dimensionality of the distance signal is still very large. Therefore, we adopt PCA ${ }^{11}$ to find transformation for dimensionality reduction. PCA is a classical linear approach to reduce data dimensionality and has been effectively used in face recognition ${ }^{12}$ and gait recognition ${ }^{6}{ }^{13}$. The process of PCA similar to ${ }^{6}$ is illustrated as follows:

Given $c$ classes for training and each class represents a sequence of distance signals of one person. $D_{i, j}$ is the $j$ th distance signal in class $i$ and $N_{i}$ is the number of distance signals in the $i$ th class. The total number of training samples is $N_{T}=N_{1}+N_{2}+$ $\cdots+N_{c}$ and the whole training set is represented by $\left[D_{1,1}, D_{1,2}, \cdots, D_{1, N_{1}}, D_{2,1}, \cdots, D_{c, N_{c}}\right]$. The mean $m_{d}$ of the set can be given by:

$$
m_{d}=\frac{1}{N_{T}} \sum_{i=1}^{c} \sum_{j=1}^{N_{i}} D_{i, j}
$$

The global covariance matrix $\sum$ can be represented by:

$$
\sum=\frac{1}{N_{T}} \sum_{i=1}^{c} \sum_{j=1}^{N_{i}}\left(D_{i, j}-m_{d}\right)\left(D_{i, j}-m_{d}\right)^{T}
$$

If the rank of the matrix $\sum$ is $K$, we can compute $K$ nonzero eigenvalues $\lambda_{1}, \lambda_{2}, \cdots, \lambda_{K}$ and the corresponding eigenvectors $e_{1}, e_{2}, \cdots, e_{K}$.

According to the theory of PCA, each distance signal can be approximated by taking only the $k<K$ largest eigenvalues $\lambda_{1} \geqslant \lambda_{2} \geqslant \cdots \geqslant \lambda_{k}$ and the corresponding eigenvectors $e_{1}, e_{2}, \cdots, e_{k}$. Hence, we use a threshold value $T$ to ignore the small eigenvalues and their associated eigenvectors:

$$
W_{k}=\sum_{i=1}^{k} \lambda_{i} / \sum_{i=1}^{K} \lambda_{i}>T
$$

where $W_{k}$ is the accumulated variance of the first $k$ largest eigenvalues with respect to all eigenvalues. The $k$ eigenvectors associated with the $k$ largest eigenvalues spans the transformation matrix $\left[e_{1}, e_{2}, \cdots, e_{k}\right]$. Each distance signal $D_{i, j}$ can be projected to a point $P_{i, j}$ in the $k$-dimensional eigenspace by the equation

$$
P_{i, j}=\left[e_{1}, e_{2}, \cdots, e_{k}\right]^{T} D_{i, j}
$$

It is well known that $k$ is usually much smaller than the original data dimension. Therefore, the projection can drastically reduce the dimensionality of distance signals. According to Equation (7), each gait sequence can be projected to a series of points in the eigenspace. And the projection centroid $C_{i}$ can be given by averaging all these points.

$$
C_{i}=\frac{1}{N_{i}} \sum_{j=1}^{N_{i}} P_{i, j}
$$


The unit vector of the centroid is $\frac{C_{i}}{\left\|C_{i}\right\|}$, which is represented by $u_{i}$. We call the unit vector $u_{i}$ gait feature for each gait sequence.

\section{Recognition}

Once we obtain gait features, the next step is gait recognition. In this section, we introduce three classification methods - MDA with NN or ENN, BPNN and SVM. Firstly, we present the MDA method in detail as it is the main classification method we adopt. Then, we give a brief description of the BPNN and SVM methods. It is to be noted that all the three methods use the gait features produced by Section 2 as input.

\subsection{MDA Method}

$\mathrm{MDA}^{11}$ is used to solve multiple-class classification problems. It seeks a projection that best separates data of different classes in the least-square sense. Thus, MDA can optimize the class separability. Han et al. ${ }^{7}$ and Huang et al. ${ }^{13}$ use MDA to achieve best class separability in gait recognition. In this paper, we adopt MDA as formers.

Suppose the $n k$-dimensional gait features $\left\{u_{1}, u_{2}, \cdots, u_{n}\right\}$ belong to $c$ classes. The within-class scatter matrix $S_{W}$ and the between-class scatter matrix $S_{B}$ are defined as

$$
\begin{gathered}
S_{W}=\sum_{i=1}^{c} S_{i}, \\
S_{B}=\sum_{i=1}^{c} n_{i}\left(m_{i}-m\right)\left(m_{i}-m\right)^{T}
\end{gathered}
$$

where $S_{i}=\sum_{u \in D_{i}}\left(u-m_{i}\right)\left(u-m_{i}\right)^{T}, m_{i}=\frac{1}{n_{i}} \sum_{u \in D_{i}} u$, and $m=\frac{1}{n} \sum_{u \in D} u, D_{i}$ is the training set that belongs to the $i$ th class and $n_{i}$ is the number of samples in $D_{i}$. The purpose of MDA training is to maximize distances between different classes and minimize distances within each class, that is, to seek a transformation matrix $W$ that maximize the function given by

$$
J(W)=\frac{\left|\widetilde{S}_{B}\right|}{\left|\widetilde{S}_{W}\right|}=\frac{\left|W^{T} S_{B} W\right|}{\left|W^{T} S_{W} W\right|}
$$

In fact, $J(W)$ is maximized when the columns of $W$ are the generalized eigenvectors that correspond to the largest eigenvalues in

$$
S_{B} w_{i}=\lambda_{i} S_{w} w_{i}
$$

Thus, we can obtain no more than $c-1$ nonzero eigenvalues and the corresponding eigenvectors $v_{1}, v_{2}, \cdots, v_{c-1}$ to form a transformation matrix. The final feature vector $F_{i}$ for each gait sequence is obtained from the $k$-dimensional gait feature $u_{i}$ :

$$
F_{i}=\left[v_{1}, v_{2}, \cdots, v_{c-1}\right]^{T} u_{i}
$$

After the MDA training process, gait features are transformed to a new space where it become easier to classify gait features belonging to different classes.

Nevertheless, we still need classification method to obtain the final recognition results. In this paper, we choose two simple classification methods - NN and ENN. In NN test, each gait sequence is classified to the same class with its nearest neighbor. In ENN test, each gait sequence is classified to the same class with its nearest exemplar which is defined as the mean of final feature vectors for one given person in training set.

Let $G$ represent a testing gait sequence, we can compute the final feature vector $F_{G}$ according to Section 2 and Section 3.1. $G$ is classified to $\omega_{k}$ when

$$
d\left(F_{G}, F_{k}\right)=\min _{i=1}^{c} d\left(F_{G}, F_{i}\right)
$$

\subsection{BPNN Method}

Neural networks ${ }^{11}{ }^{14}$, which have been widely used in image and signal processing ${ }^{15} 16$, are very effective for solving multiple-class classification problems. Many researchers have successfully applied neural networks to face/gait recognition ${ }^{17} 181920$. $\mathrm{Chau}^{18}$ notes that neural networks facilitate gait recognition because of their highly flexible, inductive, and non-linear modeling ability. In this paper, we use one classical type of neural networks $\mathrm{BPNN}^{21}$

BPNN usually has input and output layers, with some hidden layers in between. Actually, BPNN can be likened to a flexible mathematical function which 
has many configurable internal parameters ${ }^{18}$. In order to accurately represent the complicated relationships among gait variables, these internal parameters need to be adjusted through training process.

In training process, gait features and corresponding labels are input to the network, which iteratively self-adjusts to accurately classify as many gait features as possible. Training is complete when some criterion is satisfied (e.g., interaction times reach a preset value or prediction error falls below a preset threshold).

Once the neural network is trained, we can use it to predict the gait features of testing gait sequences. It is to be noted that the trained neural network simply performs function evaluation using the internal parameters established during training process to produce an output.

\subsection{SVM Method}

The theory of SVM is based on the idea of structural risk minimization ${ }^{22}$. In many applications, SVM has been introduced as a powerful tool for solving classification problems ${ }^{23} 2425$. Consequently, many researchers have used SVM on gait recognition ${ }^{2} 1926$. However, it is to be noted that SVM is fundamentally a two-class classifier.

SVM first maps the training samples into a highdimension space (typically much higher than the original data space) and then finds a separating hyperplane that maximizes the margin between two classes in this high-dimension space. Maximizing the margin is a quadratic programming $(\mathrm{QP})$ problem and can be solved from its dual problem by introducing Lagrangian multipliers. Without any knowledge of the mapping, the SVM can find the optimal hyperplane by using the dot product functions in original space that are called kernels. There are several kernels proposed by researchers. Here, we use radial basis function (RBF). Once the optimal hyperplane is established, we can directly use a decision function to classify testing samples.

For solving multi-class problems, various methods have been proposed for combining multiple twoclass SVMs in order to build a multi-class classifier, such as "one-against-one" and "one-againstrest" methods. In this paper, we use the "one- against-one" method ${ }^{27}$ in which $k(k-1) / 2$ classifiers are constructed and each one trains samples from two different classes. In classification, we use a voting strategy: each two-class SVM is considered as a voter (i.e. $k(k-1) / 2$ voters in all), and then each testing sample is classified to the class with maximum number of votes.

\section{Experiments}

\subsection{Gait Database}

In our experiments, we use the CASIA Gait Database (Dataset B) ${ }^{28}$ which is one of the largest gait databases in gait-research community currently. The database consists of 124 subjects ( 93 males and 31 females) captured from 11 view angles (ranging from $0^{\circ}$ to $180^{\circ}$, with view angle interval of $18^{\circ}$ ). The frame size is $320 \times 240$ pixels, and the frame rate is $25 \mathrm{fps}$. There are six normal walking sequences for each subject per view. We use gait sequences numbered from 001 to 100 (subject ID, i.e., 100 subjects) of view angle $90^{\circ}$ in Dataset B to carry out our experiments. Because each subject has six normal walking sequences, we assign three sequences to training set and the remaining three sequences to testing set. Fig. 5 shows three images in this gait database.
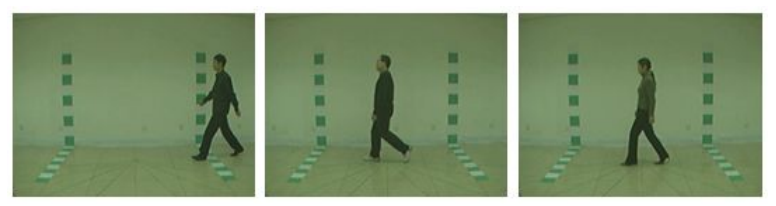

Fig. 5. Three images in CASIA Gait Database (Dataset B) with view angle $90^{\circ}$.

\subsection{Gait Feature Extraction}

In our experiments, each gait sequence is firstly preprocessed and converted into a sequence of distance signals as described in Section 2.1 and 2.2. Then, for training set, distance signals of 30 successive frames of each subject are chosen for PCA training, and eventually 47 eigenvectors corresponding to the largest 47 eigenvalues (computed according 
to the threshold $T=0.99$ ) are kept to form the transformation matrix. Finally, the gait features of both training and testing sequences are computed using the method described in Section 2.3.

\subsection{Experimental Results}

\subsubsection{MDA Results}

MDA training is carried out on all the gait features of training sequences to form a transformation matrix. Then the gait features are projected to a new eigenspace according to MDA projection Equation (13). Actually, the points projected to the new space are the final feature vectors of the training sequences. Fig. 6 shows the distribution of 15 final feature vectors belonging to five subjects respectively. For visualization, only the first threedimensional eigenspace is used. The points with the same shape belong to the same subject. From Fig. 6, we can see that these final feature vectors can be separated easily.

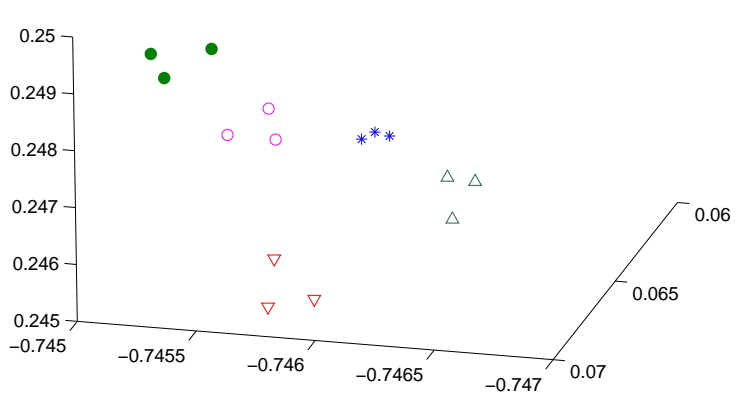

Fig. 6. The distribution of 15 final feature vectors belonging to five subjects (only the first three-dimensional eigenspace is used for visualization).

For each testing sequence, we firstly compute the gait feature by the feature extraction method described in Section 2. Then we compute the final feature vector by directly using the MDA projection Equation (13). Finally, we use NN or ENN classifier to classify the testing set.
The CCRs (Correct Classification Rate) are shown in Table 1. We compute the CCRs by four strategies: directly using $\mathrm{NN}$ and ENN on the gait feature data; and using NN and ENN on the final feature vector data produced by MDA projection.

Table 1. CCRs of the four strategies.

\begin{tabular}{lc}
\hline Recognition Methods & CCR(\%) \\
\hline NN & 72.33 \\
ENN & 69.00 \\
MDA+NN & $\mathbf{9 6 . 6 7}$ \\
MDA+ENN & $\mathbf{9 7 . 6 7}$ \\
\hline
\end{tabular}

Fig. 7 shows the cumulative match scores for rank from 1 to 50 of the four strategies. It is to be noted that the cumulative match scores of Rank $=1$ is equivalent to the CCRs as shown in Table 1 .

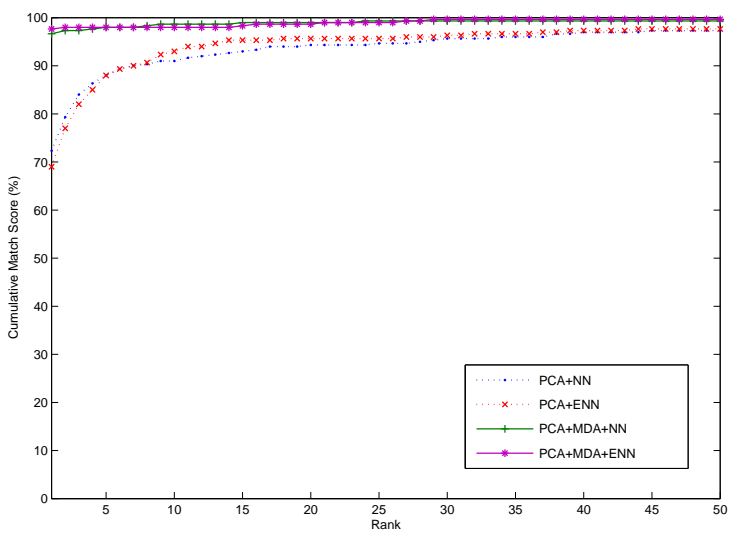

Fig. 7. Cumulative match score of the four strategies.

For completeness, we also estimate FAR (False Acceptance Rate) and FRR (False Rejection Rate) in verification mode. The ROC (Receiver Operating Characteristic) curves are shown in Fig. 8, from which we can see that the EERs (Equal Error Rate) are approximately $16 \%, 11 \%, 8 \%$ and $5 \%$ for $\mathrm{NN}$, 
ENN, MDA+NN and MDA+ENN, respectively.

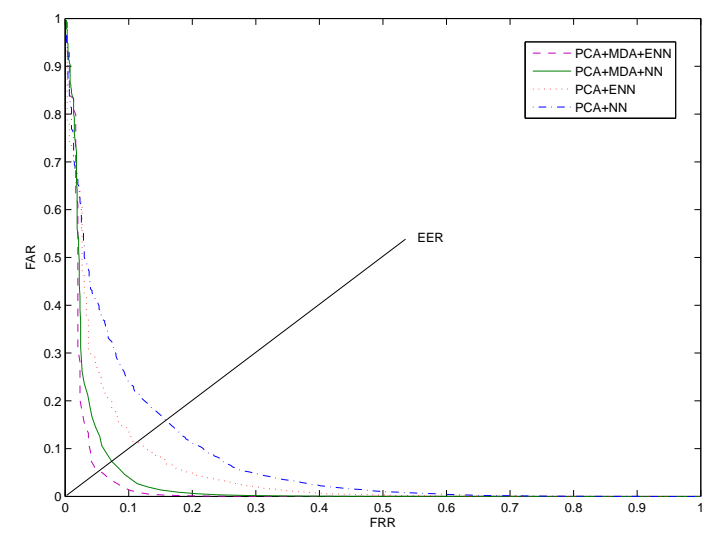

Fig. 8. ROC curves of the four strategies.

\subsubsection{BPNN and SVM Results}

In order to verify the effectiveness and robustness of our proposed feature extraction method, we also test recognition performance using BPNN and SVM classifiers.

In training process, unlike MDA which learns a transformation matrix and projects gait features to a new space, both BPNN and SVM learn a discrimination function which can be directly used to classify the testing sequences. Testing results of BPNN and SVM methods are shown in Table 2.

Table 2. CCRs of BPNN and SVM methods.

\begin{tabular}{lc}
\hline Recognition Methods & CCR(\%) \\
\hline BPNN & $\mathbf{9 4 . 3 3}$ \\
SVM & $\mathbf{9 4 . 6 7}$ \\
\hline
\end{tabular}

From Table 1 and Table 2, we can conclude that: (1) The outermost contour is discriminative, and our feature extraction method is effective; (2) The three classification approaches - MDA with NN or ENN, BPNN and SVM have similar high CCR, which demonstrates our proposed feature extraction method is robust.

\subsubsection{Comparison}

In this section, we compare the performance of the proposed method with two typical model-free methods $^{6} 28$.

In paper ${ }^{6}$, Wang et al. propose a feature extraction method based on outer contour. This method needs to unwrap the outer contour and to normalize the extracted distance signals, which is complicated and difficult to implement. And they use the NLPR database to carry out their experiments. The NLPR database contains 20 subjects and each subject has four sequences. In their experiments, three sequences are assigned to training set and the remaining one is assigned to testing set.

In paper ${ }^{28}$, a feature extraction method based on gait energy image is applied, and the CASIA Gait Database (Dataset B) is used for experiments. The CASIA Gait Database (Dataset B) contains 124 subjects and each subject has six normal walking sequences. In their experiments, four sequences are assigned to training set and the other two sequences are assigned to testing set.

In our experiments, we test our proposed method on a subdatabase of the CASIA Gait Database (Dataset B) containing 100 subjects. And we assign three sequences to training set and the other three sequences to testing set as described in Section 4.1.

The CCRs of the three different gait recognition methods are shown in Table 3. It is to be noted that the CCRs are compared on side view database (i.e. view angle $90^{\circ}$ ). Although some experiment conditions of the three methods are different, the comparison result can reflect the excellent performance of our method to some extent.

Table 3. CCRs of the three recognition methods.

\begin{tabular}{lc}
\hline Recognition Methods & Best CCR(\%) \\
\hline Wang $^{6}$ & 75.00 \\
Yu $^{28}$ & 97.60 \\
Our Method & $\mathbf{9 7 . 6 7}$ \\
\hline
\end{tabular}

\section{Conclusions}

In this paper, we propose a novel and simple gait recognition method based on outermost contour. An 
adaptive silhouette extraction algorithm and a series of postprocessing is applied to segment and normalize all frames of each gait sequence. Then, after carrying out the proposed feature extraction method based on outermost contour, we perform PCA to reduce the dimensionality of the distance signals derived from the outermost contours of silhouette images and then compute gait feature for each gait sequence. Three classification methods - MDA with NN or ENN, BPNN, and SVM are used for recognition. Experimental results show that all these three approaches can achieve similar high accuracy which indicates the outermost contour feature is robust and our feature extraction method is effective. The best accuracy $97.67 \%$ achieved in this paper and the comparisons with the state-of-the-art gait recognition methods demonstrate that our proposed method is a very encouraging gait recognition method in gait recognition community.

\section{Acknowledgments}

We would like to express our thanks to the Institute of Automation, Chinese Academy of Sciences for CASIA Gait Database. This work is partly supported by National Natural Science Foundation of China under Grant No. 61070097 and Natural Science Foundation of Shandong Province under Grant No. Z2008G05 and ZR2009GM003.

\section{References}

1. M. S. Nixon, J. N. Carte, J. M. Nash, P. S. Huang, D. Cunado and S. V. Stevenage, "Automatic gait recognition," Proc. Biometrics Personal Identification in Networked Society, 231-219 (1999).

2. L. Lee and W. E. L. Grimson," "Gait Analysis for Recognition and Classification," The 5th IEEE Intl. Conf. on Automatic Face and Gesture Recognition, Washington, DC, USA, 148-155 (2002).

3. C. Y. Yam, M. S. Nixon, and J. N. Carter "Gait Recognition By Walking and Running: A Model-Based Approach," The 5th Asian Conference on Computer Vision(ACCV), Melbourne, Australia, 1-6 (2002).

4. H. Murase and R. Sakai, "Moving object recognition in eigenspace representation: gait analysis and lip reading," Pattern Recognition Letters, 17 (2), 155-162 (1996).
5. J. J. Little and J. E. Boyd, "Recognizing People by Their Gait: The Shape of Motion," Videre: J. Computer Vision Research, 1 (2), 1-32 (1998).

6. L. Wang, T. Tan, H. Ning and W. Hu, "Silhouette Analysis-Based Gait Recognition for Human Identification," IEEE Trans. on Pattern Analysis and Machine Intelligence, 25 (12), 1505-1518 (2003).

7. J. Han and B. Bhanu, "Individual Recognition Using Gait Energy Image,’ IEEE Trans. on Pattern Analysis and Machine Intelligence, 28 (2), 316-322(2006).

8. C. Chen, J. Liang, H. Zhao, H. Hu and J. Tian, "Frame difference energy image for gait recognition with incomplete silhouettes," Pattern Recognition Letters, 30, 977-984 (2009).

9. L. Liu, Y. Yin and W. Qin, "Gait recognition based on outermost contour," Proc. Intl. Conf. on Rough Set and Knowledge Technology, 395-402 (2010).

10. C. Fu, P. Li, Y. Wen, H. Yuan and B. Ye, "Gait Sillhouette Extraction Algorithm Using Gauss Model," Chinese journal of sensors and actuators, 21 (7), (2008).

11. R. O. Duda, P. E. Hart and D. G. Stork, Pattern Classification, Second Edition, Wiley (2000).

12. P. N. Belhumeur, J. P. Hespanha and D. J. Kriegman, "Eigenfaces versus Fisherfaces: Recognition Using Class Specific Linear Projection," IEEE Trans. on Pattern Analysis and Machine Intelligence, 19 (7), 711-720 (1997).

13. P. S. Huang, C. J. Harris and M. S. Nixon, "Recognising Humans by Gait via Parameteric Canonical Space," Artificial Intelligence in Engineering, 13, 359-366 (1999).

14. T. M. Mitchell, Machine Learning, McGraw Hill (1997).

15. M. Egmont-Petersen, D. Ridder and H. Handels, "Image Processing with Neural Networksła Review," Pattern Recognition, 35, 2279-2301 (2002).

16. Y. H. Hu and J. N. Hwang, Handbook of Neural Network Signal Processing, CRC Press (2002).

17. N. K. Narayanan, V. Kabeer, "Face Recognition Using Nonlinear Feature Parameter and Artificial Neural Network," International Journal of Computational Intelligence Systems, 3 (5), 566-574 (2010).

18. T. Chau, "A Review of Analytical Techniques for Gait Data. Part 2: Neural Network and Wavelet Methods," Gait and Posture, 13 (2), 102-120 (2001).

19. E. Zhang, J. Lu and G. Duan, "Gait Recognition via Independent Component Analysis Based on Support Vector Machine and Neural Network," Lecture Notes in Computer Science, 3610, 640-649 (2005).

20. J. H. Yoo, D. Hwang, K. Y. Moon and M. S. Nixon, "Automated Human Recognition by Gait using Neural Network," Image Processing Theory, Tools and Applications, 1-6 (2008)

21. R. Hecht-Nielsen, "Theory of the Backpropagation Neural Network," Int. Joint Con. on Neural Networks, 
1, 593-605 (1989)

22. V. N. Vapnik, The Nature of Statistical Learning Theory, Springer-Verlag, New York (1995).

23. T. S. Furey, N. Cristianini, N. Duffy, D. W. Bednarski, M. Schummer and D. Haussler, "Support Vector Machine Classification and Validation of Cancer Tissue Samples Using Microarray Expression Data," Bioinformatics, 15 (10), 906-914 (2000).

24. X. Xu, R. Law and T. Wu, "Support Vector Machines with Manifold Learning and Probabilistic Space Projection for Tourist Expenditure Analysis," International Journal of Computational Intelligence Systems, 2 (1), 17-26 (2009).

25. B. Yao, C. Yang, J. Yao and J. Sun, "Tunnel Surrounding Rock Displacement Prediction Using Support
Vector Machine," International Journal of Computational Intelligence Systems, 3 (6), 843-852 (2010).

26. R. K. Begg, M. Palaniswami, Br. Owen, "Support Vector Machines for Automated Gait Classification," IEEE Transactions on Biomedical Engineering, 52 (5), 828-838 (2005).

27. S. Knerr, L. Personnaz, and G. Dreyfus, "Single-layer learning revisited: a stepwise procedure for building and training a neural network," In J. Fogelman, editor, Neurocomputing: Algorithms, Architectures and Applications, Springer-Verlag (1990).

28. S. Yu, D. Tan, T. Tan, "A Framework for Evaluating the Effect of View Angle, Clothing and Carrying Condition on Gait Recognition," The 18th Intl. Conf. on Pattern Recognition, 441-444 (2006). 